\section{Appearance concerns in ophthalmic patients}

\begin{abstract}
Aims This study aimed to determine the psychosocial and appearance-related concerns of a sample of ophthalmic patients by measuring a range of psychological, social, and demographic factors.

Methods Standardized psychological measures including anxiety, depression, appearance-related distress, self-discrepancy, appearance salience and valence were administered to 98 participants attending ophthalmic outpatient clinics in either London, Bristol, Sheffield or Bradford. Differences between groups were explored using $t$-tests and ANOVA, relationships between all variables were investigated using
\end{abstract} Pearson's correlation coefficient.

Results Although mean scores for psychological adjustment were within the normal range, some participants were experiencing considerable levels of generalized anxiety. Being older, male, and married or living with a partner was related to significantly better adjustment. Better adjustment was also related to a less visible area of concern, greater disguisability of the affected area, a more positive evaluation of their own appearance, less engagement in comparing themselves with others, greater feelings of being accepted by others, appearance being less important to their selfconcept, and a smaller discrepancy between the persons ideal and actual appearance. Conclusions A majority of ophthalmic patients adjust positively to the demands placed on them. By identifying the variables that are associated with successful adaptation, the specific psychological interventions and appropriate systems of support can be put in place to help those who are adversely affected. Eye (2011) 25, 1039-1044; doi:10.1038/eye.2011.116; published online 20 May 2011

Keywords: disfigurement; psychological distress; outpatients
H James ${ }^{1}$, E Jenkinson², R Harrad ${ }^{3}$, DG Ezra ${ }^{4}$ and S Newman', members of the Appearance Research Collaboration (ARC) ${ }^{5}$

\section{Introduction}

Patients with a wide variety of ophthalmic conditions often report concerns about the appearance of their eyes, as well as issues relating to functional deficits. ${ }^{1}$ The psychosocial impact of disfiguring eye conditions has been well documented, with $10-45 \%$ of outpatients experiencing clinical anxiety, between 3 and $18 \%$ clinical depression, and between 45 and $46 \%$ raised levels of appearance-related distress and social avoidance. ${ }^{1}$ Approximately $80 \%$ of patients with strabismus have been found to attribute problems in their personal life to their squint, with all patients adversely affected by the 'cosmetic blemish of squint' and reporting problems making and maintaining friends. ${ }^{2,3}$ Avoidance, concealment, and behaviours indicating self-consciousness such as reduced eye contact, eye rubbing, abnormal head posture, dark glasses, staying at home, and avoiding situations that bring attention to their eye have also been described.

Nevertheless, there is significant variability in the ability of patients to cope with the challenges of a disfiguring eye condition, and researchers have begun the task of identifying predictors of psychosocial adjustment. Contrary to the expectations of the lay public and many health care providers, important findings from research, clinical practice, and personal accounts suggest that the extent, type, and severity of a disfigurement are not consistently strong predictors of adjustment; however, the visibility of the condition may exacerbate distress. ${ }^{4}$ There is a consensus amongst researchers and practitioners that individual adjustment is affected by a complex interplay of physical, socio-cultural, and psychosocial factors, ${ }^{4-7}$ in which some factors contribute to distress, whereas others seem to 'buffer' a person against the stresses and strains of living with a disfigurement.

A number of factors have been thought to have a positive impact on psychosocial coping for patients with eye conditions. This includes
${ }^{1}$ Health Services Research, City University, London, UK

${ }^{2}$ Centre of Appearance Research, UWE, Bristol, UK

${ }^{3}$ Bristol Eye Hospital, Bristol, UK

${ }^{4}$ Moorfields Eye Hospital and UCL Institute of Ophthalmology NIHR Biomedical Research Centre for Ophthalmology, London, UK

Correspondence: S Newman, Health Services Research, School of Community \& Health Sciences, City University, 20 Bartholomew Close, London EC1A 7QN, USA. Tel: + 44 (0)207 040 5829; Fax: + 44 (0)207 0400875 E-mail: stanton.newman.1 @city.ac.uk

${ }^{5}$ Nichola Rumsey, James Byron-Daniel, Rodger Charlton, Alex Clarke, Sally-Ann Clarke, Diana Harcourt, Hayley James, Elizabeth Jenkinson, Antje Lindenmeyer, Tim Moss, Rob Newell, Stanton Newman, Krysia Saul, Andrew Thompson, Eleanor Walsh, Paul White, and Emma Williams

Received: 25 January 2011 Accepted in revised form: 28 March 2011 Published online: 20 May 2011 
advancing age, ${ }^{8}$ perceived social support, and levels of concern about appearance issues. Research from ophthalmic outpatient clinics reveal that higher anxiety levels are significantly related to greater worry about appearance, belief that the condition is more noticeable to others and to less favourable perception of social support. Higher levels of depression were related to a greater worry about appearance and lower perceived social support. ${ }^{1}$

This study aims to take this work further by using a range of validated psychosocial measures to identify the psychosocial and appearance-related concerns of a range of disfiguring ophthalmic conditions. The variables included in this study aim to extend previous work, by measuring a range of psychological, as well as social and demographic factors that may predict psychological adjustment. Identifying these predictors will facilitate the development of targeted psychosocial interventions and enable recommendations to be made regarding the provision of psychological support in eye clinics. In addition, this study includes outpatients from several geographic locations London, Bristol, Bradford, and Sheffield.

\section{Materials and methods}

A total of 98 adult patients attending one of four ophthalmic outpatient clinics in London, Bristol, and Sheffield or an ocular prosthetics clinic in London between 2007 and 2008 were recruited. All patients included in the study presented to the clinic with eye conditions, which affected the appearance of their eyes. Such conditions include ptosis, thyroid eye disease, strabismus, ocular cancer, and trauma.

\section{Materials}

The two measures of psychological well being included were the DAS24 and the HADS. The other intervening cognitive variables were selected on the basis that they are considered potentially modifiable through psychosocial intervention and from the experience of clinicians and research are associated with the extent of psychological adjustment.

\section{The questionnaire included six validated scales}

- The Hospital Anxiety and Depression Scale (HADS), ${ }^{9}$ a valid and reliable 14-item self-screening questionnaire for depression and anxiety in patients with physical health problems. Higher scores indicate greater levels of depression or anxiety and scores
$11>$ on either of the HADS subscales indicates clinical caseness, which were the individual to be examined by an experienced mental health professional, it is highly likely that they would be diagnosed to be suffering from an identifiable psychiatric disorder. The HADS has shown adequate internal consistency over a range of studies and good concurrent validity when compared with a range of other anxiety and depression scales $(r=0.60$ to 0.80$) .{ }^{10}$ It has been used to good effect in studies with patients with facial disfigurements. ${ }^{11}$

- The Derriford Appearance Scale short form (DAS24), ${ }^{12}$ a shortened version of the DAS59 ${ }^{13}$ measures appearance-related distress and dysfunction. It has been widely used in research related to disfigurement. Total scores range from 11 to 96 with lower scores representing lower levels of distress. It has adequate internal consistency $(\alpha=0.92)$, test retest reliability $(r=0.82)$, concurrent validity with the DAS59 $(r=0.88)$ and convergent validity with measures of anxiety, depression, social avoidance, social distress, fear of negative evaluation, negative effect, and shame $(r>0.45)$.

- Physical Appearance Discrepancy Questionnaire (PADQ) based on the work of Altabe and Thompson (1996), ${ }^{14}$ assesses how different the participant feels they look from their ideal, as considered by themselves, the media and friends and family. Scores range from 4 to 28 with higher scores representing a greater discrepancy.

- The Valence of Appearance scale (CARVAL) ${ }^{15}$ measures how positively or negatively the participant evaluates their own appearance, with higher scores indicating a more positive evaluation. Scores range from 6 to 36 .

- The Salience of Appearance scale (CARSAL) ${ }^{15}$ measures the extent to which appearance is important to a person. Higher scores indicate greater salience. Scores range from 6 to 36 .

- The Iowa-Netherlands Comparison Orientation measures $(\mathrm{INCOM})^{16}$ measures the individual differences in how often a person compares their appearance to that of others. Higher scores indicate a higher frequency of comparisons with others on the basis of appearance. Scores range from 11 to 55 . The authors cite good psychometric properties of the scale.

Participants were asked to state the area of the body they were most concerned about and asked to rate from 1 (extremely easy) to 7 (impossible) how difficult the participant felt it was to hide or disguise the aspect of appearance about which they were most concerned. Participants were also asked to rate their feelings of social acceptance; the extent to which the respondent felt accepted by their social group and society in general. 


\section{Statistical analysis}

Differences between groups were explored using $t$-tests and ANOVA. The relationships between all variables were investigated using Pearson's product-moment correlation coefficient. All tests were two tailed, with a significance level of $P=0.05$. The data were analysed using SPSS version 14 (SPSS Inc., Chicago, IL, USA).

\section{Statement of ethics}

We certify that all applicable institutional and governmental regulations concerning the ethical use of human volunteers were followed during this research.

\section{Results}

The mean age of participants was 52.79 years ranging from 18 to 87 . In all, $62 \%$ were female and $81 \%$ were white. Approximately $58 \%$ were married or lived with their partner. Having a disease or illness $(16.3 \%)$ and getting older $(16.3 \%)$ were the two main self-reported causes of the condition leading to appearance concern. For $79 \%$ of participants, the eyes were their main area of concern in regards to their appearance.

Table 1 displays the mean scores for all variables. The DAS24, anxiety, and depression mean scores were within the normal range. However, standard deviations and ranges indicate that the variation in scores between participants was considerable with some patients experiencing considerable levels of generalized anxiety. The distribution of patients with anxiety and depression, which was classified as either 'normal', 'moderate' or 'caseness' is illustrated in Figure 1.

The independent sample $t$-test showed no significant differences between men and women on scores of depression, visibility, and social acceptance. There were significant differences on the DAS24 $\mathrm{t}(93)=3.808$, $P=0.000$ (two-tailed), anxiety $\mathrm{t}(93)=2.282, P=0.025$ (two-tailed), disguisability $\mathrm{t}(83)=2.159, P=0.034$, salience $\mathrm{t}(93)=3.482, P=0.001$, social comparison $\mathrm{t}(50.47)=2.201, P=0.032$, and valence $\mathrm{t}(93)=3.515$, $P=0.001$ with males scoring lower on all variables (Table 1). However, the effect size ranged from very small (0.008) to large (0.139).

A significant negative correlation was found between age and the DAS24 $r=-0.33, n=95, P<0.01$ and salience $r=-0.22, n=95, P=0.03$, with older participants experiencing less distress and dysfunction, as a result of their appearance and considered appearance to be less important.

The relationships between the DAS24, HADS, and all other variables are displayed in Table 2. The DAS24 was correlated significantly with all other variables, in the
Table 1 Descriptive statistics for sample

\begin{tabular}{|c|c|c|c|}
\hline Group & $\mathrm{N}$ & Mean & $S D$ \\
\hline \multicolumn{4}{|l|}{ DAS24 } \\
\hline Overall & 98 & 37.80 & 13.03 \\
\hline Female & 62 & 41.11 & 12.97 \\
\hline Male & 34 & 31.10 & 10.56 \\
\hline \multicolumn{4}{|l|}{ Depression } \\
\hline Overall & 98 & 4.44 & 3.31 \\
\hline Female & 62 & 4.84 & 3.60 \\
\hline Male & 34 & 3.55 & 2.51 \\
\hline \multicolumn{4}{|l|}{ Anxiety } \\
\hline Overall & 98 & 7.16 & 4.57 \\
\hline Female & 62 & 7.86 & 4.70 \\
\hline Male & 34 & 5.66 & 4.04 \\
\hline \multicolumn{4}{|l|}{ Visibility } \\
\hline Overall & 94 & 5.39 & 2.17 \\
\hline Female & 59 & 5.58 & 2.05 \\
\hline Male & 33 & 5.00 & 2.40 \\
\hline \multicolumn{4}{|c|}{ Disguisability } \\
\hline Overall & 88 & 4.61 & 1.70 \\
\hline Female & 58 & 4.90 & 1.69 \\
\hline Male & 28 & 4.07 & 1.65 \\
\hline \multicolumn{4}{|c|}{ Self-discrepancy } \\
\hline Overall & 96 & 30.08 & 10.77 \\
\hline Female & 61 & 32.62 & 9.52 \\
\hline Male & 33 & 25.13 & 11.63 \\
\hline \multicolumn{4}{|l|}{ Salience } \\
\hline Overall & 98 & 33.22 & 6.80 \\
\hline Female & 62 & 34.88 & 6.50 \\
\hline Male & 34 & 30.20 & 6.54 \\
\hline \multicolumn{4}{|c|}{ Social Comparison } \\
\hline Overall & 98 & 35.72 & 6.89 \\
\hline Female & 62 & 36.89 & 5.67 \\
\hline Male & 34 & 33.34 & 8.39 \\
\hline \multicolumn{4}{|c|}{ Social acceptance } \\
\hline Overall & 97 & 11.65 & 2.61 \\
\hline Female & 61 & 11.52 & 2.49 \\
\hline Male & 34 & 12.06 & 2.70 \\
\hline \multicolumn{4}{|l|}{ Valence } \\
\hline Overall & 98 & 21.95 & 7.66 \\
\hline Female & 62 & 23.89 & 7.43 \\
\hline Male & 34 & 18.35 & 7.04 \\
\hline
\end{tabular}

expected directions. The largest correlations were found between the DAS24 and overall discrepancy and valence. Depression and anxiety were found to significantly correlate with overall discrepancy and valence, ranging from 0.24 to 0.43 . Small significant correlations were also found between anxiety and visibility and anxiety and salience. 


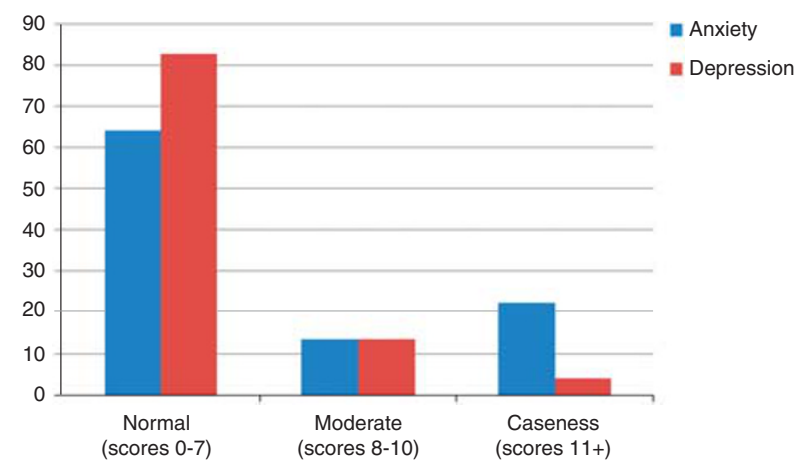

Figure 1 Classification of anxiety and depression by percentage.

\section{Discussion}

This study aimed to determine the psychosocial and appearance-related concerns of a sample of ophthalmic patients. Scores on the DAS24 suggest that participants were experiencing distress and dysfunction in relation to their appearance, with scores higher than that of the general population ${ }^{17}$ but lower than previous studies of patients attending ophthalmic outpatient clinics. ${ }^{1,18}$ Interestingly, nearly $40 \%$ of participants reported levels of distress and dysfunction in relation to their appearance that were higher than population norms. This confirms previous findings in ophthalmic outpatients with disfiguring eye disease ${ }^{1}$ and suggests that this is a pervasive issue for patients, which could be an important motivating factor for consulting with ophthalmologists and surgeons.

Similarly, although mean scores for anxiety and depression suggest that many participants were in the normal range, these mean scores were higher than that of a non-clinical sample, ${ }^{19}$ and similar to those of preoperative strabismus patients ${ }^{18}$ but lower than previous reports of other ophthalmic outpatient clinics dealing disfiguring disease. ${ }^{1,20}$ Although the majority of participants fell below the clinical cut-off scores on the HADS, over $22 \%$ of the population displayed 'caseness' levels of anxiety. This is slightly lower than previously reported $^{1}$ but is nevertheless indicative of a high level of unmet need in this population.

Although female participants were found to experience greater levels of general anxiety, reported higher levels of distress and dysfunction in relation to their appearance, placed more value on their appearance, compared their appearance more often with others and evaluated their appearance more negatively than males, the differences in mean scores were marginal. An exception to this pattern is in relation to appearancerelated distress and dysfunction, in which large
Table 2 Pearson's correlation coefficients between outcomes and all other variables

\begin{tabular}{lccc}
\hline & DAS & Depression & Anxiety \\
\hline Visibility & $0.22^{*}$ & 0.12 & 0.20 \\
Disguisability & $0.31^{*}$ & 0.13 & 0.15 \\
Salience & $0.42^{*}$ & 0.12 & $0.27^{*}$ \\
Social Comparison & $0.34^{*}$ & 0.03 & 0.15 \\
Overall discrepancy & $0.54^{*}$ & $0.43^{*}$ & $0.31^{*}$ \\
Social Acceptance & $-0.48^{*}$ & -0.11 & -0.15 \\
Valence & $0.55^{*}$ & $0.37^{*}$ & $0.24^{*}$ \\
\hline${ }^{*}<<0.05$ & & &
\end{tabular}

differences between men and women were reported. This is consistent with the disfigurement literature. ${ }^{21}$

As it has been found in studies involving clinical patients and the general population, ${ }^{17,21}$ older age was related to appearance being less important and lower levels of appearance-related distress and dysfunction. These higher levels reported by the younger responders may be a reflection of the perception that appearance is considered more important for relationships and social activity, hence the significantly higher scores for appearance salience in this study.

The correlations between adjustment, visibility, and disguisability suggest that those patients who perceive their disfigurement to be highly visible and experience difficulties disguising this feature, exhibit increased levels of general anxiety and appearance-related distress and dysfunction. This is in line with previous research that suggests that participants who believe their disfiguring condition is more noticeable to other people are more likely to experience increased levels of anxiety. ${ }^{1}$

Our study is limited by the fact that data on clinical diagnosis were not collected and therefore analysis looking at the impact of specific conditions was not possible. It is feasible that other chronic conditions and their resultant symptoms and treatment may have impacted upon psychological adjustment, future research would therefore benefit from capturing this data. Furthermore, participants are those attending for hospital appointments and therefore actively seeking treatment. As highlighted in a recent review ${ }^{22}$ this may be because these patients are experiencing greater levels of appearance-related distress, have worse visual function or because they or their primary care provider is unaware of the treatment options. Further research is needed to identify what type of patient seeks treatment for disfiguring eye conditions.

These findings should be interpreted by clinicians with some caution as there was considerable variability in scores from patients indicating that it is not always the case that markers such as being male, older, with a less 
visible and more disguisable condition will buffer a patient from distress about their appearance. Surgical decision making and assessment for psychological support for example, should still consider each patient's concerns and expectations on a case by case basis, rather than relying on gender, age, visibility, or perceived disguisability of the condition as reliable indicators of unmet need.

In summary, this study found that although many participants were coping successfully with concerns about their appearance, there were substantial numbers of patients experiencing high levels of distress and dysfunction in relation to their appearance. This study also identified a number of psychosocial variables related to adjustment including the importance placed on appearance, how a person views their own appearance, feelings of social acceptance, how often a person compares their appearance with others, and the discrepancy between how a person feels they look in reality compared with their ideal self. These factors help us better understand how patients positively adjust to their disfiguring eye condition. These findings are of clinical importance as they offer an opportunity for clinical intervention and are now being used to develop structured psychological interventions to improve successful psychological adjustment and address the unmet needs of ophthalmic outpatients with disfiguring conditions.

\section{Summary}

\section{What was known before}

- Patients with disfiguring eye conditions can experience significant psychological distress and report using avoidant and concealing behaviours to hide their altered appearance. Important findings from research and clinical findings suggest that the extent, type, and severity of a disfigurement do not consistently predict levels of psychological adjustment. Individual psychological adjustment is affected by a complex interplay of physical, socio-cultural, and psychosocial factors, in which some factors contribute to distress, whereas others seem to 'buffer' a person against the stresses and strains of living with a disfigurement.

\section{What this study adds}

- Although a majority of ophthalmic patients adjusted positively to the demands placed on them, there are a proportion of people who are adversely affected. A number of demographic and psychosocial variables were associated with better psychological adjustment. By identifying these variables, some of which are amenable to change, interventions can be designed to help those who are affected.

\section{Conflict of interest}

The authors declare no conflict of interest.

\section{Acknowledgements}

We would like to thank the participants for taking part in the study, as well as the clinic staff who assisted in recruitment. We would also like to acknowledge $\mathrm{Mr}$ Rundell, Miss Currie, Miss Tan, and Professor Rennie from the Ophthalmology Department at Royal Hallamshire Hospital Sheffield and Mr Hugo Henderson at the Royal Free Hospital London for assistance in recruiting patients. Our thanks also to the ARC Advisory Committee, in particular, service user representatives Olivia Giles, Pam Warren and Luke Wiseman for their input into the research programme. As well as the Healing Foundation for funding the study, in association with the Welton Foundation and the Worshipful Company of Tin Plate Workers. DE acknowledges financial support from the Department of Health through the award made by the National Institute for Health Research to Moorfields Eye Hospital NHS Foundation Trust and UCL Institute of Ophthalmology for a Specialist Biomedical Research Centre for Ophthalmology. The views expressed in this publication are those of the authors and not necessarily those of the Department of Health. Funding for the study was provided by the Healing Foundation in association with the Welton Foundation and the Worshipful Company of Tin Plate Workers.

\section{References}

1 Clarke A, Rumsey N, Collin JRO, Wyn-Williams M. Psychosocial distress associated with disfiguring eye conditions. Eye 2003; 17: 35-40.

2 Menon V, Saha J, Tandon R, Mehta M, Khokhar S. Study of the psychosocial aspects of strabismus. J Pediatr Ophthalmol Strabismus 2002; 39: 203-208.

3 Olitsky SE, Sudesh S, Graziano A, Hamblen J, Brooks SE, Shaha $\mathrm{SH}$. The negative psychosocial impact of strabismus in adults. J AAPOS 1999; 3: 209-211.

4 Rumsey N, Harcourt D. Body image \& disfigurement: issues \& interventions. Body Image 2004; 1: 83-97.

5 Moss T. Individual variation in adjusting to visible differences. In: Lansdown R, Rumsey N, Bradbury E, Carr A, Partridge J (eds). Visibly Different: Coping with Disfigurement. Butterworth Heinemann: Oxford, 1997.

6 Endriga MC, Kapp-Simon KA. Psychological issues in craniofacial care: state of the art. Cleft Palate Craniofac J 1999; 36: 3-11.

7 Clarke A. Psychosocial aspects of facial disfigurement: problems, management and the role of a lay-led organisation. Psychol Health Med 1999; 4: 127-142.

8 Nelson BA, Gunton KB, Lasker JN, Nelson LB, Drohan LA. The psychosocial aspects of strabismus in teenagers and adults and the impact of surgical correction. J AAPOS 2008; 12: 72-76.

9 Zigmond AS, Snaith RP. The Hospital Anxiety And Depression Scale. Acta Psychiat Scand 1983; 67: 361-370. 
10 Bjelland I, Dahl AA, Haug TT, Neckelmann D. The validity of the Hospital Anxiety and Depression Scale. An updated literature review. J Psychosomatic Res 2002; 52(2): 69-77.

11 Martin CR, Newell RJ. Factor structure of the hospital anxiety and depression scale in individuals with facial disfigurement. Psychol Health Med 2004; 9(3): 327-336.

12 Moss TP, Harris D, Carr T. Manual for the Derriford Appearance Scale 24 (DAS24). Musketeer Press: Bradford on Avon, 2004.

13 Carr T, Harris D, James C. The Derriford Appearance Scale (DAS-59): a new scale to measure individual responses to living with problems of appearance. Brit J Health Psych 2000; 5: 201-215.

14 Altabe M, Thompson JK. Body image: A cognitive self-schema construct? Cognit Ther Res 1996; 20: 171-193.

15 Rosser BA. Cognitive information processing biases in appearance adjustment: the role of appearance self-schema. Unpublished PhD thesis, University of the West of England 2008.

16 Gibbons FX, Buunk BP. Individual differences in social comparison: development of a scale of social comparison orientation. J Pers Soc Psychol 1999; 76: 129-142.

17 Carr T, Moss T, Harris D. The DAS24: a short form of the Derriford Appearance Scale DAS59 to measure individual responses to living with problems of appearance. Brit J Health Psych 2005; 10: 285-298.

18 Jackson S, Harrad RA, Morris M, Rumsey N. The psychosocial benefits of corrective surgery for adults with strabismus. Brit J Ophthalmol 2006; 90: 883-888.

19 Crawford JR, Henry JD, Crombie C, Taylor EP. Normative data for the HADS from a large non-clinical sample. Brit J Clin Psychol 2001; 40: 429-434.

20 Rumsey N, Clarke A, White P, Wyn-Williams M, Garlick W. Altered body image: appearance-related concerns of people with visible disfigurement. J Adv Nurs 2004; 48: 443-453.

21 Harris D, Carr A. Prevalence of concern about physical appearance in the general population. Brit J Plast Surg 2001; 54: 223-226.

22 Durnian JA, Noonan CP, Marsh IP. The psychosocial effects of adult strabismus: a review. Br J Ophthalmol 2011; 95: 450-453. 